\title{
Robustness and sensitivity analysis of risk measurement procedures*
}

\section{Rama $\mathrm{CONT}^{1,2}$, Romain DEGUEST ${ }^{1}$ and Giacomo SCANDOLO ${ }^{3}$}

1) IEOR Dept, Columbia University, New York.

2) Laboratoire de Probabilités et Modèles Aléatoires,

CNRS- Université de Paris VI-VII, France.

3) Dipartimento di Matematica per le Decisioni, Università di Firenze, Italia.

Revised version of: Report No. 2007-06

Center for Financial Engineering, Columbia University.

\begin{abstract}
Measuring the risk of a financial portfolio involves two steps: estimating the loss distribution of the portfolio from available observations and computing a "risk measure" which summarizes the risk of the portfolio. We define the notion of "risk measurement procedure", which includes both of these steps and introduce a rigorous framework for studying the robustness of risk measurement procedures and their sensitivity to changes in the data set. Our results point to a conflict between subadditivity and robustness of risk measurement procedures and show that the same risk measure may exhibit quite different sensitivities depending on the estimation procedure used. Our results illustrate in particular that using recently proposed risk measures like CVaR/ expected shortfall lead to a less robust risk measurement procedure than historical Value at Risk. We also propose alternative risk measurement procedures which possess the robustness property.
\end{abstract}

Keywords: robust statistics, coherent risk measures, law invariant risk measures, Value at Risk, expected shortfall.

*We thank Hans Föllmer, Peter Bank, Paul Embrechts, Gerhard Stahl and seminar participants at QMF 2006 (Sydney), Humboldt University (Berlin), Torino, Lecce, INFORMS Applied Probability Days (Eindhoven), Cornell ORIE seminar and the Harvard Statistics Seminar for helpful comments. This project has benefited from partial funding by the European Research Network "Advanced Mathematical Methods for Finance" (AMAMEF). 


\section{Contents}

1 Introduction $\quad 3$

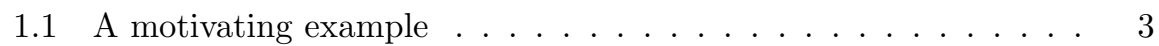

1.2 Contribution of the present work . . . . . . . . . . . . 4

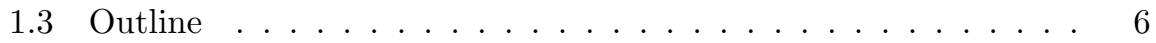

2 Estimation of risk measures $\quad 6$

2.1 Risk measures . . . . . . . . . . . . . . . . . . 7

2.2 Estimation of risk measures . . . . . . . . . . . . . 8

2.2.1 Historical risk estimators . . . . . . . . . . . . . . . 9 9

2.2.2 Maximum likelihood estimators . . . . . . . . . . . . 10

2.3 Effective risk measures . . . . . . . . . . . . . . . 11

3 Qualitative robustness 13

$3.1 \mathcal{C}$-robustness of a risk estimator . . . . . . . . . . . . . 13

3.2 Qualitative robustness of historical risk estimators . . . . . . . 14

3.2.1 Historical $\mathrm{VaR}_{\alpha}$.................. 15

3.2.2 Historical estimator of ES and spectral risk measures . . 15

3.2.3 A robust family of risk estimators . . . . . . . . . 16

3.3 Qualitative robustness of maximum likelihood risk estimator . . 16

4 Sensitivity analysis 18

4.1 Historical VaR . . . . . . . . . . . . . . . . . . . . 18

4.2 Historical estimators of Expected Shortfall and spectral risk measures . . . . . . . . . . . . . . . . . 19

4.3 ML risk estimators for Gaussian distributions . . . . . . . . . . . 20

4.4 ML risk estimators for Laplace distributions . . . . . . . . . . . . 21

4.5 Finite sample effects . . . . . . . . . . . . . . . 22

5 Discussion $\quad 22$

5.1 Summary of main results . . . . . . . . . . . . . . . . . 22

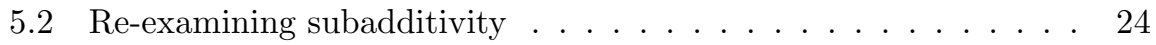

5.3 Beyond distribution-based risk measures . . . . . . . . . . . 24

A Proofs $\quad \mathbf{2 5}$

A.1 Proof of Proposition $2 \ldots \ldots \ldots \ldots . \ldots \ldots$

A.2 Proof of Theorem 2 . . . . . . . . . . . . . 25 


\section{Introduction}

One of the main purposes of quantitative modeling in finance is to quantify the risk of financial portfolios. In connection with the widespread use of Valueat-Risk and related risk measurement methodologies and the Basel committee guidelines for risk-based requirements for regulatory capital, methodologies for measuring of the risk of financial portfolios have been the focus of recent attention and have generated a considerable theoretical literature $[1,2,3,7,8,9]$. In this theoretical approach to risk measurement, a risk measure is represented as a map assigning a number (a measure of risk) to each random payoff. The focus of this literature has been on the properties of such maps and requirements for the risk measurement procedure to be coherent, in a static or dynamic setting.

Since most risk measures such as Value-at-Risk or Expected Shortfall are defined as functionals of the portfolio loss distribution, an implicit starting point is the knowledge of the loss distribution. In applications, however, this probability distribution is unknown and should be estimated from (historical) data as part of the risk measurement procedure. Thus, in practice, measuring the risk of a financial portfolio involves two steps: estimating the loss distribution of the portfolio from available observations and computing a risk measure which summarizes the risk of this loss distribution. While these two steps have been considered and studied separately, they are intertwined in applications and an important criterion in the choice of a risk measure is the availability of a method for accurately estimating it. Estimation or mis-specification errors in the portfolio loss distribution can have a considerable impact on risk measures, and it is important to examine the sensitivity of risk measures to these errors [10].

\subsection{A motivating example}

Consider the following example, based on a data set of 1000 loss scenarios for a derivatives portfolio incorporating hundreds of different risk factors. ${ }^{1}$ The historical Value-at-Risk (VaR) i.e. the quantile of the empirical loss distribution, and the Expected Shortfall [1] of the empirical loss distribution, computed at $99 \%$ level are, respectively, $8.887 \mathrm{M} \$$ and $9.291 \mathrm{M} \$$.

To examine the sensitivity of these estimators to a single observation in the data set, we compute the (relative) change (in \%) in the estimators when a new observation is added to the data set. Figure 1 displays this measure of sensitivity as a function of the size of the observation added. While the levels of the two risk measures are not very different, they display quite different sensitivities to a change in the data set, the Expected Shortfall being much more sensitive to large observations while VaR has a bounded sensitivity. While Expected Shortfall has the advantage of being a coherent risk measure [1, 3], it appears to lack robustness with respect to small changes in the data set.

Another point, which has been left out of most studies on risk measures (with

\footnotetext{
${ }^{1}$ Data courtesy of Société Générale Risk Management unit.
} 
the notable exception of [10]) is the impact of the estimation method on these sensitivity properties. A risk measure such as Expected Shortfall (ES) can be estimated in different ways: either directly from the empirical loss distribution ("historical ES") or by first estimating a parametric model (Gaussian, Laplace etc.) from the observed sample and computing the Expected Shortfall using the estimated distribution. Figure 2 shows the sensitivity of the Expected Shortfall

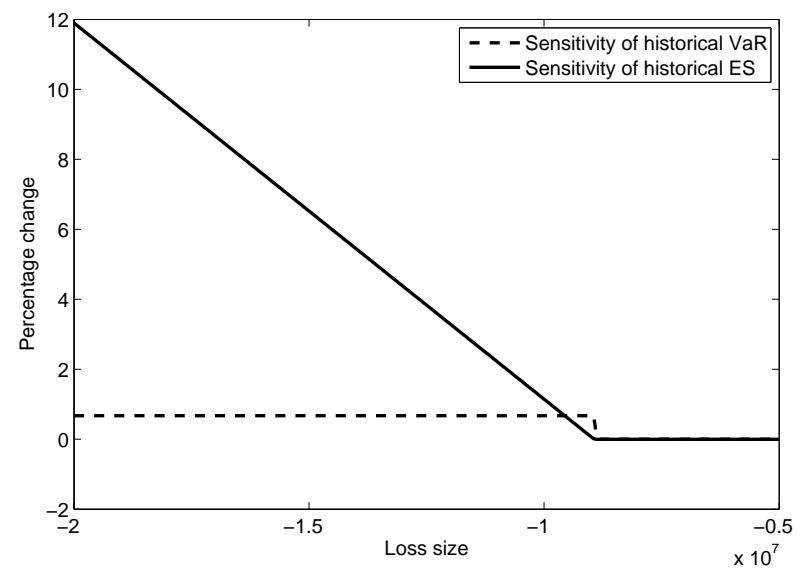

Figure 1: Empirical sensitivity (in percentage) of the historical $\mathrm{VaR}_{99 \%}$ and historical $\mathrm{ES}_{99 \%}$.

for the same portfolio as above, but estimated using three different methods. We observe that different estimators for the same risk measure exhibit very different sensitivities to an additional observation (or outlier).

These examples motivate the need for assessing the sensitivity and robustness properties of risk measures in conjunction with the estimation method being used to compute them. In order to study the interplay of a risk measure and its estimation method used for computing it, we define the notion of risk measurement procedure, as a two-step procedure which associates to a payoff $X$ and a data set $D_{n}$ of size $n$ a risk estimate $\widehat{\rho}(X)$ for $X$ based on the data set $D_{n}$. This estimator of the "theoretical" risk measure $\rho(X)$ is said to be robust if small variations in the loss distribution -resulting either from estimation or mis-specification errors- result in small variations in the estimator.

\subsection{Contribution of the present work}

In the present work, we propose a rigorous approach for examining how estimation issues can affect the computation of risk measures, with a particular focus on robustness and sensitivity analysis of risk measurement procedures, using tools from robust statistics $[11,13]$. By contrast with the considerable literature on risk measures $[1,2,3,7,15,16,17]$, which does not discuss estimation 


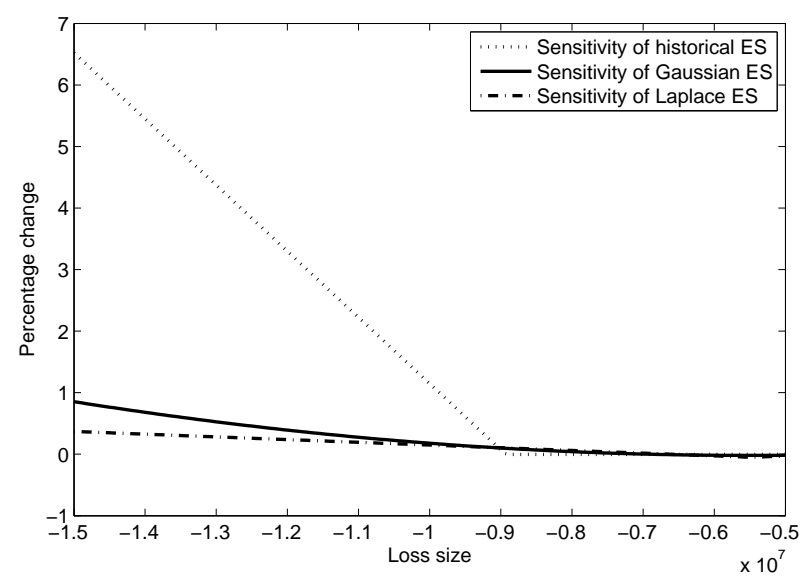

Figure 2: Empirical sensitivity (in percentage) of the $\mathrm{ES}_{\alpha=1 \%}$ estimated with different methods.

issues, we argue that the choice of the estimation method and the risk measure should be considered jointly using the notion of risk estimator.

We introduce a qualitative notion of 'robustness' for a risk measurement procedure and a way of quantifying it via sensitivity functions. Using these tools we show that there is a conflict between coherence (more precisely, the sub-additivity) of a risk measure and the robustness, in the statistical sense, of its commonly used estimators. This consideration goes against the traditional arguments for the use of coherent risk measures merits discussion. We complement this abstract result by computing measures of sensitivity, which allow to quantify the robustness of various risk measures with respect to the data set used to compute them. In particular, we show that the same "risk measure" may exhibit quite different sensitivities depending on the estimation procedure used. These properties are studied in detail for some well known examples of risk measures: Value-at-Risk, Expected Shortfall/ CVaR [1, 16, 17] and the class of spectral risk measures introduced by Acerbi [2]. Our results illustrate in particular that historical Value-at-Risk, while failing to be sub-additive, leads to a more robust procedure than alternatives such as Expected shortfall.

Statistical estimation and sensitivity analysis of risk measures have also been studied by Gourieroux \& Liu [10] and Heyde et al. [12]. Gourieroux \& Liu [10] nonparametric estimators of distortion risk measures (which includes the class studied in this paper) and focuses on the asymptotic distribution of these estimators. By contrast we study their robustness and sensitivity using tools from robust statistics. Heyde et al. [12], which appeared simultaneously with the first version of this paper, contains some ideas similar to ours but in a finite data set (i.e. non-asymptotic) framework. We show that, using appropriate definitions of consistency and robustness, the discussion can be extended to a large-sample/asymptotic framework which is the usual setting for discussion of 
estimators and also enables to establish a clear link between properties of risk estimators and those of risk measures.

\subsection{Outline}

Section 2 recalls some basic notions on distribution-based risk measures and establishes the distinction between a risk measure and a risk measurement procedure. We show that a risk measurement procedure applied to a data set can be viewed as the application of an effective risk measure to the empirical distribution obtained from this data and give examples of effective risk measures associated to various risk estimators.

Section 3 defines the notion of robustness for a risk measurement procedure and examines whether this property holds for commonly used risk measurement procedures. We show in particular that there exists a conflict between the subadditivity of a risk measure and the robustness of its estimation procedure.

In section 4 we define the notion of sensitivity function for a risk measure and compute sensitivity functions for some commonly used risk measurement procedures. In particular we show that, while historical VaR has a bounded sensitivity to a change in the underlying data set, the sensitivity of Expected Shortfall estimators is unbounded. We discuss in section 5 some implications of our findings for the design of risk measurement procedures in finance.

\section{Estimation of risk measures}

Let $(\Omega, \mathcal{F}, \mathbb{P})$ be a given probability space representing market outcomes and $L^{0}$ be the space of all random variables. We denote by $\mathcal{D}$ the (convex) set of cumulative distribution functions (cdf) on $\mathbb{R}$. The distribution of a random variable $X$ is denoted $F_{X} \in \mathcal{D}$. and we write $X \sim F$ if $F_{X}=F$. The Lévy distance [13] between two $\operatorname{cdf} F, G \in \mathcal{D}$ is:

$$
d(F, G) \triangleq \inf \{\varepsilon>0: F(x-\varepsilon)-\varepsilon \leq G(x) \leq F(x+\varepsilon)+\varepsilon \forall x \in \mathbb{R}\},
$$

The upper and lower quantiles of $F \in \mathcal{D}$ of order $\alpha \in(0,1)$ are defined, respectively, by:

$$
q_{\alpha}^{+}(F) \triangleq \inf \{x \in \mathbb{R}: F(x)>\alpha\} \geq q_{\alpha}^{-}(F) \triangleq \inf \{x \in \mathbb{R}: F(x) \geq \alpha\} .
$$

Abusing notation, we denote $q_{\alpha}^{ \pm}(X)=q_{\alpha}^{ \pm}\left(F_{X}\right)$. For $p \geq 1$ we denote by $\mathcal{D}^{p}$ the set of distributions having finite $p$-th moment, i.e.

$$
\int_{\mathbb{R}}|x|^{p} d F(x)<\infty ;
$$

and by $\mathcal{D}_{-}^{p}$ the set of distributions whose left tail has finite $p$-moment. We denote $\mu(F)$ the mean of $F \in \mathcal{D}^{1}$ and $\sigma^{2}(F)$ the variance of $F \in \mathcal{D}^{2}$. For any $n \geq 1$ and any $\mathbf{x}=\left(x_{1}, \ldots, x_{n}\right) \in \mathbb{R}^{n}$,

$$
F_{\mathbf{x}}^{\mathrm{emp}}(x) \triangleq \frac{1}{n} \sum_{i=1}^{n} I_{\left\{x \geq x_{i}\right\}}
$$


denotes the empirical distribution of the data set $\mathbf{x} ; \mathcal{D}_{\text {emp }}$ will denote the set of all empirical distributions.

\section{$2.1 \quad$ Risk measures}

The "Profit and Loss" (P\&L) or payoff of a portfolio over a specified horizon may be represented as a random variable $X \in L \subset L^{0}(\Omega, \mathcal{F}, \mathbb{P})$, where negative values for $X$ correspond to losses. The set $L$ of such payoffs is assumed to be a convex cone containing all constants. A risk measure on $L$ is a map $\rho: L \rightarrow \mathbb{R}$ assigning to each P\&L $X \in L$ a number representing its degree of riskiness.

Artzner et al [3] advocated the use of coherent risk measures, defined as follows.

Definition 1 (Coherent risk measure, [3]). A risk measure $\rho: L \rightarrow \mathbb{R}$ is coherent if it is:

1. Monotone (decreasing). $\rho(X) \leq \rho(Y)$ provided $X \geq Y$

2. Cash-additive (additive with respect to cash reserves). $\rho(X+c)=\rho(X)-c$ for any $c \in \mathbb{R}$.

3. Positive homogeneous. $\rho(\lambda X)=\lambda \rho(X)$ for any $\lambda \geq 0$ :

4. Subadditive. $\rho(X+Y) \leq \rho(X)+\rho(Y)$

The vast majority of risk measures used in finance are statistical, or distributionbased risk measures, i.e. they depend on $X$ only through its distribution $F_{X}$ :

$$
F_{X}=F_{Y} \Rightarrow \rho(X)=\rho(Y)
$$

In this case $\rho$ can be represented as a map on the set of probability distributions, which we still denote by $\rho$. Therefore, by setting

$$
\rho\left(F_{X}\right) \triangleq \rho(X),
$$

we can view see $\rho$ as a map defined on (a subset of) the set of probability distributions $\mathcal{D}$.

We focus on the following class of distribution-based risk measures, introduced in [1] and [15], which contains all examples used in the literature:

$$
\rho_{m}(X)=-\int_{0}^{1} q_{u}^{-}(X) m(d u),
$$

where $m$ is a probability measure on $(0,1)$. Let $\mathcal{D}_{m}$ be the set of distributions of r.v. for which the above risk measure is finite. $\rho_{m}$ can then be viewed as a map $\rho_{m}: \mathcal{D}_{m} \mapsto \mathbb{R}$. Notice that if the support of $m$ does not contain 0 nor 1 , then $\mathcal{D}_{m}=\mathcal{D}$.

Three cases deserve particular attention: 
Value at Risk (VaR) This is the risk measure which is the most used in practice and corresponds to the choice $m=\delta_{\alpha}$ for a fixed $\alpha \in(0,1)$ (usually $\alpha \leq 10 \%$ ), that is

$$
\operatorname{VaR}_{\alpha}(F) \triangleq-q_{\alpha}^{-}(F)
$$

Its domain of definition is all $\mathcal{D}$

Expected shortfall (ES) It corresponds to choosing $m$ as the uniform distribution over $(0, \alpha)$, where $\alpha \in(0,1)$ is fixed:

$$
\mathrm{ES}_{\alpha}(F) \triangleq \frac{1}{\alpha} \int_{0}^{\alpha} \operatorname{VaR}_{u}(F) d u .
$$

In this case, $\mathcal{D}_{m}=\mathcal{D}_{-}^{1}$, the set of distributions having integrable left tail.

Spectral risk measures $[1,2]$ This class of risk measures generalizes ES and corresponds to choosing $m(d u)=\phi(u) d u$, where $\phi:[0,1] \rightarrow[0,+\infty)$ is a density on $[0,1]$ and $u \mapsto \phi(u)$ is decreasing. Therefore:

$$
\rho_{\phi}(F) \triangleq \int_{0}^{1} \operatorname{VaR}_{u}(F) \phi(u) d u .
$$

If $\phi \in L^{q}(0,1)$ (but not in $L^{q+\varepsilon}$ ) and $\phi \equiv 0$ around 1 , then $\mathcal{D}_{m}=\mathcal{D}_{-}^{p}$, where $p^{-1}+q^{-1}=1$

For any choice of the weight $m, \rho_{m}$ defined in (1) is monotone, additive with respect to cash and positive homogeneous. The subadditivity of such risk measures has been characterized as follows $[2,7,15]$ :

Proposition $1([2,7,15])$. The risk measure $\rho_{m}$ defined in (1) is sub-additive (hence coherent) on $\mathcal{D}_{m}$ if and only if it is a spectral risk measure.

As a consequence, we recover the well known facts that ES is a coherent risk measure, while VaR is not.

\subsection{Estimation of risk measures}

Once a (distribution-based) risk measure $\rho$ has been chosen, in practice one has first to estimate the P\&L distribution of the portfolio from available data and then apply the risk measure $\rho$ to this distribution. This can be viewed as a two-step procedure:

1. Estimation of the loss distribution $F_{X}$ : one can use either an empirical distribution obtained from a historical or simulated sample or a parametric form whose parameters are estimated from available data. This step can be formalized as a function from $\mathcal{X}=\cup_{n \geq 1} \mathbb{R}^{n}$, the collection of all possible datasets, to $\mathcal{D}$; if $\mathbf{x} \in \mathcal{X}$ is a dataset, we denote $\widehat{F}_{\mathbf{x}}$ the corresponding estimate of $F_{X}$. 
2. Application of the risk measure $\rho$ to the estimated P\&L distribution $\widehat{F}_{\mathbf{x}}$, which yields an estimator $\widehat{\rho}(\mathbf{x}) \triangleq \rho\left(\widehat{F}_{\mathbf{x}}\right)$ for $\rho(X)$.

We call the combination of these two steps the risk measurement procedure:

Definition 2 (Risk measurement procedure). A risk measurement procedure $(\mathrm{RMP})$ is a couple $(M, \rho)$, where $\rho: \mathcal{D}_{\rho} \rightarrow \mathbb{R}$ is a risk measure and $M: \mathcal{X} \rightarrow \mathcal{D}_{\rho}$ an estimator for the loss distribution.

The outcome of this procedure is a risk estimator $\widehat{\rho}: \mathcal{X} \rightarrow \mathbb{R}$ defined as

$$
\mathbf{x} \mapsto \widehat{\rho}(\mathbf{x}) \triangleq \rho\left(\widehat{F}_{\mathbf{x}}\right),
$$

that estimates $\rho\left(F_{X}\right)$ given the data $\mathbf{x}$ (see diagram).

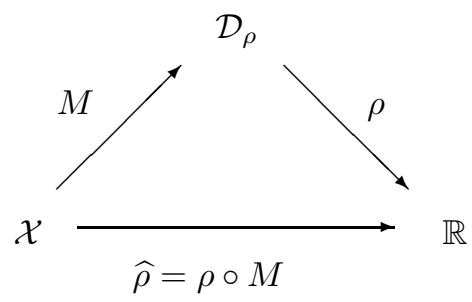

\subsubsection{Historical risk estimators}

The historical estimator $\widehat{\rho}^{h}$ associated to a risk measure $\rho$ is the estimator obtained by applying $\rho$ to the empirical P\&L distribution (sample cdf) $\widehat{F}_{\mathbf{x}}=$ $F_{\mathbf{x}}^{\text {emp: }}$

$$
\widehat{\rho}^{h}(\mathbf{x})=\rho\left(F_{\mathbf{x}}^{\mathrm{emp}}\right) .
$$

For a risk measure $\rho_{m}$, as in (1),

$$
\widehat{\rho}_{m}^{h}(\mathbf{x})=\rho_{m}\left(F_{\mathbf{x}}^{\mathrm{emp}}\right)=-\sum_{i=1}^{n} w_{n, i} x_{(i)}, \quad \mathbf{x} \in \mathbb{R}^{n},
$$

where $x_{(k)}$ is the $k$-th least element of the set $\left\{x_{i}\right\}_{i \leq n}, w_{n, i} \triangleq m\left(\frac{i-1}{n}, \frac{i}{n}\right]$ for $i=1, \ldots, n-1$, and $w_{n, n}=m\left(\frac{n-1}{n}, 1\right)$. Historical estimators are L-estimators in the sense of Huber [13].

Example 2.1. Historical $\mathrm{VaR}_{\alpha}$ is given by

$$
\widehat{\operatorname{VaR}}_{\alpha}^{h}(\mathbf{x})=-x_{(\lfloor n \alpha\rfloor+1)},
$$

where $\lfloor a\rfloor$ denotes the integer part of $a \in \mathbb{R}$.

Example 2.2. The historical expected shortfall $\mathrm{ES}_{\alpha}$ is given by

$$
\widehat{\mathrm{ES}}_{\alpha}^{h}(\mathbf{x})=-\frac{1}{n \alpha}\left(\sum_{i=1}^{\lfloor n \alpha\rfloor} x_{(i)}+x_{(\lfloor n \alpha\rfloor+1)}(n \alpha-\lfloor n \alpha\rfloor)\right)
$$


Example 2.3. The historical estimator of the spectral risk measure $\rho_{\phi}$ associated to $\phi$ is given by:

$$
\hat{\rho}_{\phi}^{h}(\mathbf{x})=-\sum_{i=1}^{n} w_{n, i} x_{(i)}, \quad \text { where } \quad w_{n, i}=\int_{(i-1) / n}^{i / n} \phi(u) d u .
$$

\subsubsection{Maximum likelihood estimators}

In the parametric approach to loss distribution modeling, a parametric model is assumed for $F_{X}$ and parameters are estimated from data using, for instance, maximum likelihood. We call the risk estimator obtained "maximum likelihood risk estimator" (MLRE). We discuss these estimators for scale families of distributions, which include as a special case (although a multidimensional one) the common variance-covariance method for VaR estimation.

Let $F$ be a centered distribution. The scale family generated by a reference distribution $F$ is defined by

$$
\mathcal{D}_{F} \triangleq\{F(\cdot \mid \sigma): \sigma>0\} \quad \text { where } \quad F(x \mid \sigma) \triangleq F\left(\frac{x}{\sigma}\right) .
$$

If $F \in \mathcal{D}^{p}(p \geq 1)$, then $\mathcal{D}_{F} \subset \mathcal{D}^{p}$ and it is common to choose $F$ with mean 0 and variance 1 , so that $F(\cdot \mid \sigma)$ has mean 0 and variance $\sigma^{2}$. In line with common practice in short-term risk management we assume that the risk factors changes have zero mean. Two examples of scale families of distributions that we will study are:

- the Gaussian family where $F$ has density

$$
f(x)=\frac{1}{\sqrt{2 \pi}} \exp \left(-\frac{x^{2}}{2}\right) .
$$

- the Laplace or double exponential family where $F$ has density

$$
f(x)=\frac{1}{2} \exp (-|x|) .
$$

The Maximum Likelihood Estimator (MLE) $\widehat{\sigma}=\widehat{\sigma}^{\mathrm{mle}}(\mathbf{x})$ of $\sigma$ is defined by

$$
\widehat{\sigma}=\arg \cdot \max _{\sigma>0} \sum_{i=1}^{n} \log f\left(x_{i} \mid \sigma\right)
$$

and solves following non-linear equation

$$
\sum_{i=1}^{n} x_{i} \frac{f^{\prime}\left(\frac{x_{i}}{\sigma}\right)}{f\left(\frac{x_{i}}{\sigma}\right)}=-n \sigma
$$

Let $\rho$ be a positively homogeneous risk measure. Then

$$
\rho(F(\cdot \mid \sigma))=\sigma \rho(F)
$$


If the scale parameter is estimated by maximum likelihood, the associated risk estimator of $\rho$ is then given by

$$
\widehat{\rho}^{\mathrm{mle}}(\mathbf{x})=c \widehat{\sigma}^{\mathrm{mle}}(\mathbf{x})
$$

Example 2.4 (MLRE for a Gaussian family).

The MLE of the scale parameter in the Gaussian scale family is

$$
\widehat{\sigma}(\mathbf{x})=\sqrt{\frac{1}{n} \sum_{i=1}^{n} x_{i}^{2}} .
$$

The resulting risk estimators are given by $\widehat{\rho}(\mathbf{x})=c \widehat{\sigma}(\mathbf{x})$ where, depending on the risk measure considered, $c$ is given by

$$
\begin{aligned}
& c=\operatorname{VaR}_{\alpha}(F)=-z_{\alpha} \\
& c=\operatorname{ES}_{\alpha}(F)=\frac{\exp \left\{-z_{\alpha}^{2} / 2\right\}}{\alpha \sqrt{2 \pi}} \\
& c=\rho_{\phi}(F)=-\int_{0}^{1} z_{u} \phi(u) d u .
\end{aligned}
$$

where $z_{\alpha}$ is the $\alpha$-quantile of a standard normal distribution.

Example 2.5 (ML risk estimators for Laplace distributions).

The MLE of the scale parameter in the Laplace scale family is

$$
\widehat{\lambda}(\mathbf{x})=\frac{1}{n} \sum_{i=1}^{n}\left|x_{i}\right|
$$

Note that this scale estimator is not the standard deviation but the Mean Absolute Deviation (MAD). The resulting risk estimator is

$$
\widehat{\rho}(\mathbf{x})=c \widehat{\lambda}(\mathbf{x})
$$

where $c$ takes the following values, depending on the risk measure considered (we assume $\alpha \leq 0.5$ ):

$$
\begin{aligned}
& c=\operatorname{VaR}_{\alpha}(F)=-\log (2 \alpha) \\
& c=\operatorname{ES}_{\alpha}(F)=-\log (2 \alpha-1) \\
& c=\rho_{\phi}(F)=-\int_{0}^{1 / 2} \log (2 u) \phi(u) d u+\int_{1 / 2}^{1} \log (2-2 u) \phi(u) d u .
\end{aligned}
$$

\subsection{Effective risk measures}

In all of the above examples we observe that the risk estimator $\widehat{\rho}(x)$ computed from a data set $x=\left(x_{1}, \ldots, x_{n}\right)$ can be expressed in terms of the empirical distribution $F_{x}^{\mathrm{emp}}$; in other words there exists a risk measure $\rho_{\text {eff }}$ such that, for 
any data set $x=\left(x_{1}, \ldots, x_{n}\right)$, the risk estimator $\widehat{\rho}(x)$ is equal to the new risk measure $\rho_{\text {eff }}$ applied to the empirical distribution:

$$
\rho_{\text {eff }}\left(F_{x}^{\mathrm{emp}}\right)=\widehat{\rho}(x)
$$

We will call $\rho_{\text {eff }}$ the effective risk measure associated to the risk estimator $\widehat{\rho}$. In other words, while $\rho$ is the risk measure we are interested in computing, the effective risk measure $\rho_{\text {eff }}$ is the risk measure which the procedure actually computes.

So far the effective risk measure $\rho_{\text {eff }}$ is defined for all empirical distributions by (13). Consider now a risk estimator $\widehat{\rho}$ which is consistent with the risk measure $\rho$ at $F \in \mathcal{D}_{\rho}$, that is

$$
\widehat{\rho}\left(X_{1}, \ldots, X_{n}\right)^{n \rightarrow \infty} \rightarrow \rho(F) \quad \text { a.s. }
$$

for any IID sequence $X_{i} \sim F$. Consistency of a risk estimator for a class of distributions of interest is a minimal requirement to ask for. If $\widehat{\rho}(F)$ is consistent with the risk measure $\rho$ for $F \in \mathcal{D}_{\text {eff }} \subset \mathcal{D}_{\rho}$, we can extend $\rho_{\text {eff }}$ to $\mathcal{D}_{\text {eff }}$ as follows: for any sequence $\left(x_{n}\right)_{n \geq 1}$ such that

$$
\frac{1}{n} \sum_{k=1}^{n} \delta_{x_{n}} \stackrel{d}{\rightarrow} F \in \mathcal{D}_{\text {eff }}
$$

we define

$$
\rho_{\text {eff }}(F):=\lim _{n \rightarrow \infty} \widehat{\rho}\left(x_{1}, \ldots, x_{n}\right)
$$

Consistency guarantees that $\rho_{\text {eff }}(F)$ is independent of the chosen sequence.

Definition 3 (Effective risk measure). Let $\widehat{\rho}: \mathcal{X} \rightarrow \mathbb{R}$ be a consistent risk estimator of a risk measure $\rho$ for a class $\mathcal{D}_{\text {eff }}$ of distributions. There is a unique risk measure $\rho_{\text {eff }}: \mathcal{D}_{\text {eff }} \mapsto \mathbb{R}$ such that

- for any data set $x=\left(x_{1}, . ., x_{n}\right) \in \mathcal{X}, \rho_{\mathrm{eff}}\left(F_{x}^{\mathrm{emp}}\right)=\widehat{\rho}(x)$.

- $\rho_{\text {eff }}(F):=\lim _{n \rightarrow \infty} \widehat{\rho}\left(x_{1}, \ldots, x_{n}\right)$ for any sequence $\left(x_{n}\right)_{n \geq 1}$ such that

$$
\frac{1}{n} \sum_{k=1}^{n} \delta_{x_{n}} \stackrel{d}{\rightarrow} F \in \mathcal{D}_{\text {eff }}
$$

The equation (13) defining the effective risk measure allows in most examples to characterize $\rho_{\text {eff }}$ quite explicitly. As shown in the examples below $\rho_{\text {eff }}$ may be quite different from $\rho$ and lack many of the properties $\rho$ was initially chosen for.

Example 2.6 (Historical VaR). The empirical quantile $\widehat{\mathrm{VaR}}_{\alpha}^{h}$ is a consistent estimator of $\operatorname{VaR}_{\alpha}$ for any $F \in \mathcal{D}$ such that $q_{\alpha}^{+}(F)=q_{\alpha}^{-}(F)$. Otherwise $\widehat{\operatorname{VaR}}_{\alpha}^{h}\left(X_{1}, \ldots, X_{n}\right)$ may not have a limit as $n \rightarrow \infty$. Therefore the effective risk measure associated to $\widehat{\operatorname{VaR}}_{\alpha}^{h}$ is $\operatorname{VaR}_{\alpha}$ restricted to the set

$$
\mathcal{D}_{\text {eff }}=\left\{F \in \mathcal{D}: q_{\alpha}^{+}(F)=q_{\alpha}^{-}(F)\right\}
$$


Example 2.7 (Historical Estimator of ES and spectral risk measures). A general result on L-estimators by Van Zwet [18] implies that the historical risk estimator of any spectral risk measure $\rho_{\phi}$ (in particular of the ES) is consistent with $\rho_{\phi}$ at any $F$ where the risk measure is defined. Therefore the effective risk measure associated with $\widehat{\rho}_{\phi}^{h}$ coincides with $\rho_{\phi}$. The same remains true even if the density $\phi$ is not decreasing, so that $\rho_{\phi}$ is not a spectral risk measure.

Example 2.8 (Gaussian ML risk estimator). Consider the risk estimator introduced in Example 2.4. The associated effective risk measure is defined on $\mathcal{D}^{2}$ and given by

$$
\rho_{\text {eff }}(F)=c \sigma(F) \quad \text { where } \quad \sigma(F)=\sqrt{\int_{\mathbb{R}} x^{2} d F(x)}
$$

Example 2.9 (Laplace ML risk estimator). Consider the risk estimators introduced in Example 2.5. The associated effective risk measure is defined on $\mathcal{D}_{1}$ and given by

$$
\rho_{\mathrm{eff}}(F)=c \lambda(F) \quad \text { where } \quad \lambda(F)=\int_{\mathbb{R}}|x| d F(x)
$$

Notice that in both of these examples the effective risk measure $\rho_{\text {eff }}$ is different from the original risk measure $\rho$.

\section{Qualitative robustness}

We now define the notion of qualitative robustness of a risk estimator and use it to examine the robustness of various risk estimators considered above.

\section{1 $\mathcal{C}$-robustness of a risk estimator}

Fix a set $\mathcal{C} \subseteq \mathcal{D}$ represent the set of "plausible" loss distributions and $F \in \mathcal{C}$, assuming $F$ not an isolated point of $\mathcal{C}$, i.e. for any $\delta>0$, there exists $G \in \mathcal{C}$, with $G \neq F$, such that $d(G, F) \leq \delta$. The intuitive notion of robustness can now be made precise as follows:

Definition 4. A risk estimator $\widehat{\rho}$ is $\mathcal{C}$-robust at $F$ if for any $\varepsilon>0$ there exist $\delta>0$ and $n_{0} \geq 1$ such that, for all $G \in \mathcal{C}$ :

$$
\left.d(G, F) \leq \delta \quad \Longrightarrow \quad d\left(\mathcal{L}_{n}(\widehat{\rho}, G)\right), \mathcal{L}_{n}(\widehat{\rho}, F)\right) \leq \varepsilon \forall n \geq n_{0} .
$$

where $d$ is the Lévy distance.

In short, a (risk) estimator is $\mathcal{C}$-robust at $F$ if a small perturbation of $F$ (which stays in $\mathcal{C}$ ) results in a small change in the law of the estimators obtained from an IID sample with law $F$, i.e. the law of the estimator is continuous with respect to changes in $F$, uniformly in the size $n$ of the data set. 
When $\mathcal{C}=\mathcal{D}$, i.e. when any perturbation of the P\&L distribution is allowed, the previous definition corresponds to the notion of qualitative robustness as proposed by Huber [13]. This case is not generally interesting in econometric or financial applications since requiring robustness against all perturbations of the model $F$ is quite restrictive and excludes even estimators such as the sample mean.

Plainly, the bigger is the set of perturbations $\mathcal{C}$, the harder is for a risk estimator to be $\mathcal{C}$ robust. In the rest of this section we will assess whether the risk estimators previously introduced are $\mathcal{C}$-robust w.r.t. a suitable set of perturbations $\mathcal{C}$.

\subsection{Qualitative robustness of historical risk estimators}

The following generalization of a result of Hampel [11], is crucial for the analysis of robustness of historical risk estimators.

Proposition 2. Let $\rho$ be a risk measure and $F \in \mathcal{C} \subseteq \mathcal{D}_{\rho}$. If $\widehat{\rho}^{h}$, the historical risk estimator of $\rho$, is consistent with $\rho$ at every $G \in \mathcal{C}$, then the following are equivalent:

1. the restriction of $\rho$ to $\mathcal{C}$ is continuous (w.r.t. Lévy distance) at $F$;

2. $\widehat{\rho}^{h}$ is $\mathcal{C}$-robust at $F$.

A proof of Proposition 2 is given in the Appendix. From this Proposition, we obtain the following Corollary that provides a sufficient condition on the risk measure to ensure that the corresponding historical/empirical estimator is robust.

Corollary 1. If $\rho$ is continuous in $\mathcal{C}$ then $\widehat{\rho}^{h}$ is $\mathcal{C}$-robust at any $F \in \mathcal{C}$.

Proof. Fix $G \in \mathcal{C}$ and let $\left(X_{n}\right)_{n \geq 1}$ be an IID sequence distributed as $G$. Then, by Glivenko-Cantelli Theorem we have, for almost all $\omega$

$$
d\left(F_{\mathbf{X}(\omega)}^{\mathrm{emp}}, G\right) \rightarrow^{n \rightarrow \infty} 0, \quad \mathbf{X}=\left(X_{1}, \ldots, X_{n}\right)
$$

By continuity of $\rho$ at $G$ it holds, again for almost all $\omega$

$$
\widehat{\rho}(\mathbf{X}(\omega))=\rho\left(F_{\mathbf{X}(\omega)}^{\mathrm{emp}}\right) \rightarrow \rho(G),
$$

and therefore $\widehat{\rho}$ is consistent with $\rho$ at $G$. A simple application of Proposition 2 concludes.

Our analysis will use the following important result adapted from Huber $[13$, Theorem 3.1]. For a measure $m$ on $[0,1]$ let

$$
\mathcal{A}_{m} \triangleq\{\alpha \in[0,1]: m(\{\alpha\})>0\}
$$

the set of values where $m$ puts a positive mass. We remark that $\mathcal{A}_{m}$ is empty for a continuous $m$ (as in the definition of spectral risk measures.) 
Theorem 1. Let $\rho_{m}$ be a risk measure of the form (1). If the support of $m$ does not contain 0 nor 1 then $\rho_{m}$ is continuous at any $F \in \mathcal{D}_{\rho}$ such that $q_{\alpha}^{+}(F)=q_{\alpha}^{-}(F)$ for any $\alpha \in \mathcal{A}_{m}$. Otherwise $\rho_{m}$ is not continuous at any $F \in \mathcal{D}_{\rho}$.

In other words, a risk measure of the form (1) can be continuous at some $F$ if and only if its computation does not involve any extreme quantile (close to 0 or 1.) In this case, continuity is ensured provided $F$ is continuous at all points where $m$ has a point mass.

\subsubsection{Historical $\mathrm{VaR}_{\alpha}$}

In this case, $\mathcal{A}_{m}=\{\alpha\}$ so combining Corollary 1 and Theorem 1:

Proposition 3. The historical risk estimator of $\mathrm{VaR}_{\alpha}$ is $\mathcal{C}_{\alpha}$-robust at any $F \in \mathcal{C}_{\alpha}$, where

$$
\mathcal{C}_{\alpha} \triangleq\left\{F \in \mathcal{D}: q_{\alpha}^{+}(F)=q_{\alpha}^{-}(F)\right\} .
$$

In other words, if the quantile of the (true) loss distribution distribution is uniquely determined, then the empirical quantile is a robust estimator.

\subsubsection{Historical estimator of ES and spectral risk measures}

Let $\rho_{\phi}$ defined in (4) in terms of a density $\phi$ in $L^{q}(0,1)$, so that $\mathcal{D}_{\rho}=\mathcal{D}^{p}(p$ and $q$ are conjugate.) However, here we do not assume that $\phi$ is decreasing, so that $\rho_{\phi}$ need not be a spectral risk measure, though it is still in the form (1).

Proposition 4. For any $F \in \mathcal{D}^{p}$, the historical estimator of $\rho_{\phi}$ is $\mathcal{D}^{p}$-robust at $F$ if and only, for some $\varepsilon>0$

$$
\phi(u)=0 \quad \forall u \in(0, \varepsilon) \cup(1-\varepsilon, 1),
$$

i.e. $\phi$ vanishes in a neighborhood of 0 and 1

Proof. We have seen in paragraph 2.3 that $\widehat{\rho}_{\phi}^{h}$ is consistent with $\rho_{\phi}$ at any $F \in$ $\mathcal{D}_{\rho}$. If (15) holds for some $\varepsilon$, then the support of $m$ (recall that $m(d u)=\phi(u) d u$ ) does not contain 0 nor 1 . As $\mathcal{A}_{m}$ is empty, Theorem 1 yields continuity of $\rho$ at any distribution in $\mathcal{D}_{\rho}$. Hence, we have $\mathcal{D}_{\rho}$-robustness of $\widehat{\rho}$ at $F$ thanks to Corollary 1.

On the contrary, if (15) does not hold for any $\varepsilon$, then 0 or 1 (or both) are in the support of $m$ and therefore $\rho_{\phi}$ is not continuous at any distribution in $\mathcal{D}_{\rho}$, in particular at $F$. Therefore, by Proposition 2 we conclude that $\widehat{\rho}$ is not $\mathcal{D}_{\rho}$-robust at $F$.

An immediate, but important consequence is:

Corollary 2. The historical risk estimator of any spectral risk measure $\rho_{\phi}$ defined on $\mathcal{D}^{p}$ is not $\mathcal{D}^{p}$-robust at any $F \in \mathcal{D}^{p}$. In particular, the historical risk estimator of $E S_{\alpha}$ is not $\mathcal{D}^{1}$-robust at any $F \in \mathcal{D}^{1}$. 
Proof. It is sufficient to observe that, for a spectral risk measure, the density $\phi$ is decreasing and therefore it cannot vanish around 0 , otherwise it would vanish on the entire interval $[0,1]$.

Proposition 4 illustrates a conflict between subadditivity and robustness: as soon as we require a (distribution-based) risk measure $\rho_{m}$ to be coherent, its historical estimator fails to be robust (at least when all possible perturbations are considered).

\subsubsection{A robust family of risk estimators}

We have just seen that $\mathrm{ES}_{\alpha}$ has a non robust historical estimator. However we can remove this drawback by slightly modifyng its definition. Consider $0<\alpha_{1}<\alpha_{2}<1$ and define the risk measure

$$
\frac{1}{\alpha_{2}-\alpha_{1}} \int_{\alpha_{1}}^{\alpha_{2}} \operatorname{VaR}_{u}(F) d u
$$

This is simply the average of VaR levels across a range of loss probabilities. As

$$
\phi(u)=\frac{1}{\alpha_{2}-\alpha_{1}} I_{\left(\alpha_{1}, \alpha_{2}\right)}(u)
$$

vanishes around 0 and 1 , Proposition 1 the historical (i.e. empirical) risk estimator of this risk measure is $\mathcal{D}^{1}$ robust. Of course, the corresponding risk measure is not coherent, as $\phi$ is not decreasing. Note that for $\alpha_{1}<1 / n$ where $n$ is the sample size, this risk estimator is indistinguishable from historical Expected shortfall! Yet, unlike Expected shortfall estimators, is has good robustness properties as $n \rightarrow \infty$. One can also consider a discrete version of this risk estimator:

$$
\frac{1}{k} \sum_{j=1}^{k} \operatorname{VaR}_{u_{j}}^{h}(F) \quad 0<u_{1}<. .<u_{k}<1
$$

which enjoys similar robustness properties.

\subsection{Qualitative robustness of maximum likelihood risk es- timator}

We now discuss qualitative robustness for MLRE in a scale family of a risk measures $\rho_{m}$ defined as in (1). Let $\mathcal{D}_{F}$ be the scale family associated to the distribution $F \in \mathcal{D}$ and assume that $\mu(F)=0, \sigma(F)=1$ and $F$ admits a density $f$. Define the function

$$
\psi(x)=-1-x \frac{f^{\prime}(x)}{f(x)}, \quad x \in \mathbb{R} .
$$

The ML estimate of the scale parameter $\sigma(G)$ for $G \in \mathcal{D}_{F}$ solves

$$
\gamma(\sigma, G) \triangleq \int \psi\left(\frac{x}{\sigma}\right) G(d x)=0 \quad \text { for } \quad G \in \mathcal{D}_{F}
$$


By defining $\mathcal{D}_{\psi}=\left\{G \in \mathcal{D} \mid \int \psi(x) G(d x)<\infty\right\}$, we can extend the definition of $\sigma(G)$ to all $G \in \mathcal{D}_{\psi}$. Note that when $G \notin \mathcal{D}_{F}, \sigma(G)$ exists if $G \in \mathcal{D}_{\psi}$ but does not correspond to the ML estimate of the scale parameter of $G$. Moreover, from the definition (17), we notice that if we compute the ML estimate of the scale parameter for a distribution $F_{\mathbf{x}} \in \mathcal{D}_{\text {emp }}$ we recover the MLE $\widehat{\sigma}^{\text {mle }}(\mathbf{x})$ introduced in Equations (8) and (9). In the examples below, we have computed the function $\psi$ for the Gaussian and Laplace scale families.

Example 3.1 (Gaussian scale family).

The function $\psi^{g}$ for the Gaussian scale family is

$$
\psi^{g}(x)=-1+x^{2},
$$

and we immediately conclude that $\mathcal{D}_{\psi^{g}}=\mathcal{D}_{2}$.

Example 3.2 (Laplace scale family).

The function $\psi^{l}$ for the Laplace scale family is equal to

$$
\psi^{l}(x)=-1+|x|,
$$

and we get $\mathcal{D}_{\psi^{l}}=\mathcal{D}_{1}$.

The following result exhibits conditions on the function $\psi$ under which the MLE of the scale parameter is weakly continuous on $\mathcal{D}_{\psi}$ :

Theorem 2 (Weak continuity of the scale MLE). Let $\mathcal{D}_{F}$ be the scale family associated to the distribution $F \in \mathcal{D}$ and assume that $\mu(F)=0, \sigma(F)=1$ and $F$ admits a density $f$. Suppose now that $\psi$, defined as in (16) is even, increasing on $\mathbb{R}^{+}$, and takes values of both signs. Then, these two assertions are equivalent

- $\sigma: \mathcal{D}_{\psi} \mapsto \mathbb{R}^{+}$, defined as in (17), is weakly continuous at $F \in \mathcal{D}_{\psi}$

- $\psi$ is bounded and $\gamma(\sigma, F) \triangleq \int \psi\left(\frac{x}{\sigma}\right) F(d x)=0$ has a unique solution $\sigma=\sigma(F)$ for all $F \in \mathcal{D}_{\psi}$.

A proof is given in the Appendix. Using the above result, we can now study the qualitative robustness of parametric risk estimators for Gaussian or Laplace scale families:

Proposition 5 (Non-robustness of Gaussian and Laplace MLRE). Gaussian (resp. Laplace) MLRE of cash-additive and homogeneous risk measures are not $\mathcal{D}_{2}$-robust (resp. $\mathcal{D}_{1}$-robust) at any $F$ in $\mathcal{D}_{2}$ (resp. in $\mathcal{D}_{2}$ ).

Proof. We detail the proof for the Gaussian scale family. The same arguments hold for the Laplace scale family. Let us consider a Gaussian MLRE of a translation invariant and homogeneous risk measure, denoted by $\widehat{\rho}(\mathbf{x})=c \widehat{\sigma}(\mathbf{x})$. First of all we notice that the function $\psi^{g}$ associated to the MLE of the scale parameter of a distribution belonging to the Gaussian scale family is even, and increasing on $\mathbb{R}^{+}$. Moreover it takes values of both signs. Secondly, we recall that the effective risk measure associated to the Gaussian risk estimator is 
$\rho_{\text {eff }}(F)=c \sigma(F)$ for all $F \in \mathcal{D}_{\text {eff }}=\mathcal{D}_{\psi^{g}}=\mathcal{D}_{2}$. Therefore, as $\psi^{g}$ is unbounded, by using Theorem 2 , we know that $\rho_{\text {eff }}$ is not continuous at any $F \in \mathcal{D}_{2}$. As the Gaussian MLRE considered $\widehat{\rho}$ verifies $\widehat{\rho}(\mathbf{x})={\widehat{\rho_{\mathrm{eff}}}}^{h}(\mathbf{x})$, and is consistent with $\rho_{\text {eff }}$ at all $F \in \mathcal{D}_{2}$ by construction, we can apply Proposition 2 to conclude that, for $F \in \mathcal{D}_{2}, \widehat{\rho}$ is not $\mathcal{D}_{2}$-robust at $F$.

\section{Sensitivity analysis}

In order to quantify the degree of robustness of a risk estimator, we now introduce the concept sensitivity function:

Definition 5 (Sensitivity function of a risk estimator). The sensitivity function of a risk estimator at $F \in \mathcal{D}_{\text {eff }}$ is the function defined by

$$
S(z)=S(z ; F) \triangleq \lim _{\varepsilon \rightarrow 0^{+}} \frac{\rho_{\mathrm{eff}}\left(\varepsilon \delta_{z}+(1-\varepsilon) F\right)-\rho_{\mathrm{eff}}(F)}{\varepsilon}
$$

for any $z \in \mathbb{R}$ such that the limit exists.

$S(z, F)$ measures the sensitivity of the risk estimator to the addition of a new data point in a large sample. $S(z ; F)$ is simply the directional derivative of the effective risk measure $\rho_{\text {eff }}$ at $F$ in the direction $\delta_{z} \in \mathcal{D}$. In the language of robust statistics, this is the influence function $[4,6,11,13]$ : of the effective risk measure $\rho_{\text {eff }}$ and is related to the asymptotic variance of the historical estimator of $\rho[11,13]$.

Remark 1. If $\mathcal{D}_{\rho}$ is convex and contains all empirical distributions, then $\varepsilon \delta_{z}+$ $(1-\varepsilon) F \in \mathcal{D}_{\rho}$ for any $\varepsilon \in[0,1], z \in \mathbb{R}$ and $F \in \mathcal{D}_{\rho}$. These conditions hold for all the risk measures we are considering.

\subsection{Historical VaR}

We have seen before that the effective risk measure associated to $\widehat{\mathrm{VaR}}_{\alpha}^{h}$ is the restriction of $\mathrm{VaR}_{\alpha}$ to

$$
\mathcal{D}_{\text {eff }}=\mathcal{C}_{\alpha}=\left\{F \in \mathcal{D}: q_{\alpha}^{+}(F)=q_{\alpha}^{-}(F)\right\}
$$

The sensitivity function of the historical $\mathrm{VaR}_{\alpha}$ has a simple explicit form:

Proposition 6. If $F \in \mathcal{D}$ admits a strictly positive density $f$, then the sensitivity function at $F$ of the historical $\mathrm{VaR}_{\alpha}$ is

$$
S(z)=\left\{\begin{array}{cl}
\frac{1-\alpha}{f\left(q_{\alpha}(F)\right)} & \text { if } z<q_{\alpha}(F) \\
0 & \text { if } z=q_{\alpha}(F) \\
-\frac{\alpha}{f\left(q_{\alpha}(F)\right)} & \text { if } z>q_{\alpha}(F)
\end{array}\right.
$$


Proof. First we observe that the map $u \mapsto q(u) \triangleq q_{u}(F)$ is the inverse of $F$ and so it is differentiable at any $u \in(0,1)$ and we have:

$$
q^{\prime}(u)=\frac{1}{F^{\prime}(q(u))}=\frac{1}{f\left(q_{u}(F)\right)}
$$

Fix $z \in \mathbb{R}$ and set, for $\varepsilon \in[0,1), F_{\varepsilon}=\varepsilon \delta_{z}+(1-\varepsilon) F$, so that $F \equiv F_{0}$. For $\varepsilon>0$, the distribution $F_{\varepsilon}$ is differentiable at any $x \neq z$, with $F_{\varepsilon}^{\prime}(x)=(1-\varepsilon) f(x)>0$, and has a jump (of size $\varepsilon$ ) at the point $x=z$. Hence, for any $u \in(0,1)$ and $\varepsilon \in[0,1), F_{\varepsilon} \in \mathcal{C}_{u}$, i.e. $q_{u}^{-}\left(F_{\varepsilon}\right)=q_{u}^{+}\left(F_{\varepsilon}\right) \triangleq q_{u}\left(F_{\varepsilon}\right)$. In particular

$$
q_{\alpha}\left(F_{\varepsilon}\right)= \begin{cases}q\left(\frac{\alpha}{1-\varepsilon}\right) & \text { for } \alpha<(1-\varepsilon) F(z) \\ q\left(\frac{\alpha-\varepsilon}{1-\varepsilon}\right) & \text { for } \alpha \geq(1-\varepsilon) F(z)+\varepsilon \\ z & \text { otherwise }\end{cases}
$$

Assume now that $z>q(\alpha)$, i.e. $F(z)>\alpha$; from (21) it follows that

$$
q_{\alpha}\left(F_{\varepsilon}\right)=q\left(\frac{\alpha}{1-\varepsilon}\right), \quad \text { for } \varepsilon<1-\frac{\alpha}{F(z)} .
$$

As a consequence

$$
\begin{aligned}
S(z) & =\lim _{\varepsilon \rightarrow 0^{+}} \frac{\operatorname{VaR}_{\alpha}\left(F_{\varepsilon}\right)-\operatorname{VaR}_{\alpha}\left(F_{0}\right)}{\varepsilon}=-\left.\frac{d}{d \varepsilon} q_{\alpha}\left(F_{\varepsilon}\right)\right|_{\varepsilon=0} \\
& =-\left.\frac{d}{d \varepsilon} q\left(\frac{\alpha}{1-\varepsilon}\right)\right|_{\varepsilon=0}=-\left[\frac{1}{f\left(q\left(\frac{\alpha}{1-\varepsilon}\right)\right)} \frac{\alpha}{(1-\varepsilon)^{2}}\right]_{\varepsilon=0} \\
& =-\frac{\alpha}{f\left(q_{\alpha}(F)\right)}
\end{aligned}
$$

The case $q(\alpha)<z$ is handled in a very similar way. Finally, if $z=q(\alpha)$, then, again by $(21)$ we have $q_{\alpha}\left(F_{\varepsilon}\right)=z$ for any $\varepsilon \in[0,1)$. Hence

$$
S(z)=-\left.\frac{d}{d \varepsilon} q_{\alpha}\left(F_{\varepsilon}\right)\right|_{\varepsilon=0}=0
$$

and we conclude.

This example shows that the historical $\mathrm{VaR}_{\alpha}$ has a bounded sensitivity to a change in the data set, which means that this risk estimator is not very sensitive to a small change in the data set.

\subsection{Historical estimators of Expected Shortfall and spec- tral risk measures}

Consider a distribution $F$ having positive density $f>0$. Assume that:

$$
\int_{0}^{1} \frac{\phi(u)}{f\left(q_{u}(F)\right)} d u<\infty .
$$


Proposition 7. The sensitivity function at $F \in \mathcal{D}_{\phi}$ of the historical risk estimator of $\rho_{\phi}$ is

$$
S(z)=-\int_{0}^{1} \frac{u}{f\left(q_{u}(F)\right)} \phi(u) d u+\int_{F(z)}^{1} \frac{1}{f\left(q_{u}(F)\right)} \phi(u) d u
$$

Proof. Using the notations of the previous proof we have:

$$
\begin{aligned}
S(z) & =\lim _{\varepsilon \rightarrow 0^{+}} \int_{0}^{1} \frac{\operatorname{VaR}_{u}\left(F_{\varepsilon}\right)-\operatorname{VaR}_{u}(F)}{\varepsilon} \phi(u) d u \\
& =\int_{0}^{1} \lim _{\varepsilon \rightarrow 0^{+}} \frac{\operatorname{VaR}_{u}\left(F_{\varepsilon}\right)-\operatorname{VaR}_{u}(F)}{\varepsilon} \phi(u) d u \\
& =\int_{0}^{1}-\left[\frac{d}{d \varepsilon} q_{u}\left(F_{\varepsilon}\right)\right]_{\varepsilon=0} \phi(u) d u \\
& =\int_{0}^{F(z)} \frac{-u}{f\left(q_{u}(F)\right)} \phi(u) d u+\int_{F(z)}^{1} \frac{1-u}{f\left(q_{u}(F)\right)} \phi(u) d u,
\end{aligned}
$$

thanks to Proposition 6. We stress that changing the integral with the limit in the second equality above is legitimate. Indeed, $\lim _{\varepsilon \rightarrow 0^{+}} \varepsilon^{-1}\left(\operatorname{VaR}_{u}\left(F_{\varepsilon}\right)\right)-$ $\left.\operatorname{VaR}_{u}(F)\right)$ exists, is finite for all $u \in(0,1)$, and for $\varepsilon$ small

$$
\left|\frac{\operatorname{VaR}_{u}\left(F_{\varepsilon}\right)-\operatorname{VaR}_{u}(F)}{\varepsilon}\right|<\frac{1}{f\left(q_{u}(F)\right)} \in L^{1}(\phi),
$$

so that we can apply dominated convergence.

Since the effective risk measure associated to historical $\mathrm{ES}_{\alpha}$ is $\mathrm{ES}_{\alpha}$ itself, defined on $\mathcal{D}^{-}=\left\{F \in \mathcal{D}: \int x^{-} F(d x)<\infty\right\}$, an immediate consequence of the previous proposition is the following

Corollary 3. The sensitivity function at $F \in \mathcal{D}^{-}$for historical $E S_{\alpha}$ is

$$
S(z)= \begin{cases}-\frac{z}{\alpha}+\frac{1-\alpha}{\alpha} q_{\alpha}(F)-\mathrm{ES}_{\alpha}(F) & \text { if } z \leq q_{\alpha}(F) \\ -q_{\alpha}(F)-\mathrm{ES}_{\alpha}(F) & \text { if } z \geq q_{\alpha}(F)\end{cases}
$$

This result shows that the sensitivity of historical $\mathrm{ES}_{\alpha}$ is linear in $z$, and thus unbounded. It means that this risk measurement procedure is less robust than the historical $\operatorname{VaR}_{\alpha}$.

\subsection{ML risk estimators for Gaussian distributions}

We have seen that the effective risk measure associated to Gaussian maximum likelihood estimators of VaR, ES, or any spectral risk measure is

$$
\rho_{\text {eff }}(F)=c \sigma(F), \quad F \in \mathcal{D}_{\text {eff }}=\mathcal{D}_{2},
$$

where $c=\rho(Z), Z \sim N(0,1)$, is a constant depending only on the risk measure $\rho$ (we are not interested in its explicit value here). 
Proposition 8. The sensitivity function at $F \in \mathcal{D}_{2}$ of the Gaussian $M L$ risk estimator of a homogeneous and cash-additive risk measure $\rho$ is

$$
S(z)=\frac{\sigma c}{2}\left[\left(\frac{z}{\sigma}\right)^{2}-1\right] .
$$

Proof. Let, for simplicity, $\sigma=\sigma(F)$. Fix $z \in \mathbb{R}$ and set, as usual, $F_{\varepsilon}=(1-$ $\varepsilon) F+\varepsilon \delta_{z}(\varepsilon \in[0,1))$; observe that $F_{\varepsilon} \in \mathcal{D}^{2}$ for any $\varepsilon$. If we set $v(\varepsilon) \triangleq c \sigma\left(F_{\varepsilon}\right)$, with $c=\rho(N(0,1))$, then we have $S(z)=v^{\prime}(0)$. It is immediate to compute

$$
\begin{aligned}
\sigma^{2}\left(F_{\varepsilon}\right) & =\int_{\mathbb{R}} x^{2} F_{\varepsilon}(d x)=(1-\varepsilon) \int_{\mathbb{R}} x^{2} F(d x)+\varepsilon z^{2}-\varepsilon z^{2} \\
& =(1-\varepsilon)\left(\sigma^{2}\right)+\varepsilon z^{2}-\varepsilon^{2} z^{2}=\sigma^{2}+\varepsilon\left[z^{2}-\sigma^{2}\right]-\varepsilon^{2} \sigma^{2}
\end{aligned}
$$

As a consequence

$$
v^{\prime}(0)=c\left[\frac{d}{d \varepsilon} \sqrt{\sigma^{2}\left(F_{\varepsilon}\right)}\right]_{\varepsilon=0}=\frac{\sigma c}{2}\left[\left(\frac{z}{\sigma}\right)^{2}-1\right]
$$

\subsection{ML risk estimators for Laplace distributions}

We have seen that the effective risk measure of the Laplace MLRE of VaR, ES, or any spectral risk measure is

$$
\rho_{\mathrm{eff}}(F)=c \lambda(F), \quad F \in \mathcal{D}^{1},
$$

where $c=\rho(G), G$ is the distribution with density $g(x)=e^{-|x|} / 2$, and $\lambda(F)=$ $\int_{\mathbb{R}}|x| d F(x)$.

Proposition 9. Let $\rho$ be a translation invariant and homogeneous risk measure. The sensitivity function at $F \in \mathcal{D}^{1}$ of its Laplace MLRE is

$$
S(z)=c(|z|-\lambda(F))
$$

Proof. As usual, we have, for $z \in \mathbb{R}, S(z)=v^{\prime}(0)$, where $v(\varepsilon)=c \lambda\left(F_{\varepsilon}\right)$, $F_{\varepsilon}=(1-\varepsilon) F+\varepsilon \delta_{z}$ and $c$ is defined above. We have

$$
v(\varepsilon)=c(1-\varepsilon) \lambda(F)+c \varepsilon|z|,
$$

and we conclude that

$$
v^{\prime}(0)=c|z|-c \lambda(F)
$$

This proposition shows that the sensitivity of the Laplace MLRE at any $F \in \mathcal{D}_{1}$ is not bounded, but linear in $z$. Nonetheless, the sensitivity of the Gaussian MLRE is quadratic at any $F \in \mathcal{D}_{2}$, which indicates a higher sensitivity to outliers in the data set. 


\begin{tabular}{|c|c|}
\hline Risk estimator & Dependence in $z$ of $S(z)$ \\
\hline \hline Historical VaR & bounded \\
\hline Gaussian ML for VaR & quadratic \\
\hline Laplace ML for VaR & linear \\
\hline Historical Expected shortfall & linear \\
\hline Gaussian ML for Expected shortfall & quadratic \\
\hline Laplace ML for Expected shortfall & linear \\
\hline
\end{tabular}

Table 1: Behavior of sensitivity functions for some risk estimators.

\subsection{Finite sample effects}

The sensitivity functions computed above are valid for (asymptotically) large samples. In order to assess the finite-sample relevance accuracy of risk estimator sensitivities, we compare them with the finite-sample sensitivity

$$
S_{N}(z, F)=\frac{\widehat{\rho}\left(X_{1}, \ldots, X_{N}, z\right)-\widehat{\rho}\left(X_{1}, \ldots, X_{N}\right)}{\frac{1}{N+1}}
$$

Figure 3 compares the empirical sensitivities of historical, Gaussian, and Laplace VaR and historical, Gaussian, and Laplace ES with their theoretical (large sample) counterparts. The asymptotic and empirical sensitivities coincide for all risk estimators except for historical risk measurement procedures. For the historical ES, the theoretical sensitivity is very close to the empirical one. Nonetheless, we note that the empirical sensitivity of the historical VaR can be equal to 0 because it is strongly dependent on the integer part of $N \alpha$, where $N$ is the number of scenarios and $\alpha$ the quantile level. This dependency disappears asymptotically for large samples.

The excellent agreement shown in these examples illustrates that the expressions derived above for theoretical sensitivity functions are useful for evaluating the sensitivity of risk estimators for realistic sample sizes. This is useful since theoretical sensitivity functions are analytically computable, whereas empirical sensitivities require perturbating the data sets and recomputing the risk measures.

\section{Discussion}

\subsection{Summary of main results}

Let us now summarize the contributions and main conclusions of this study.

First, we have argued that when the estimation step is explicitly taken into account in a risk measurement procedure, issues like robustness and sensitivity to the data set are important and need to be accounted for with at least the same attention as the coherence properties set forth by Artzner et al [3]. Indeed, we do think that it is crucial for regulators and end-users to understand the 

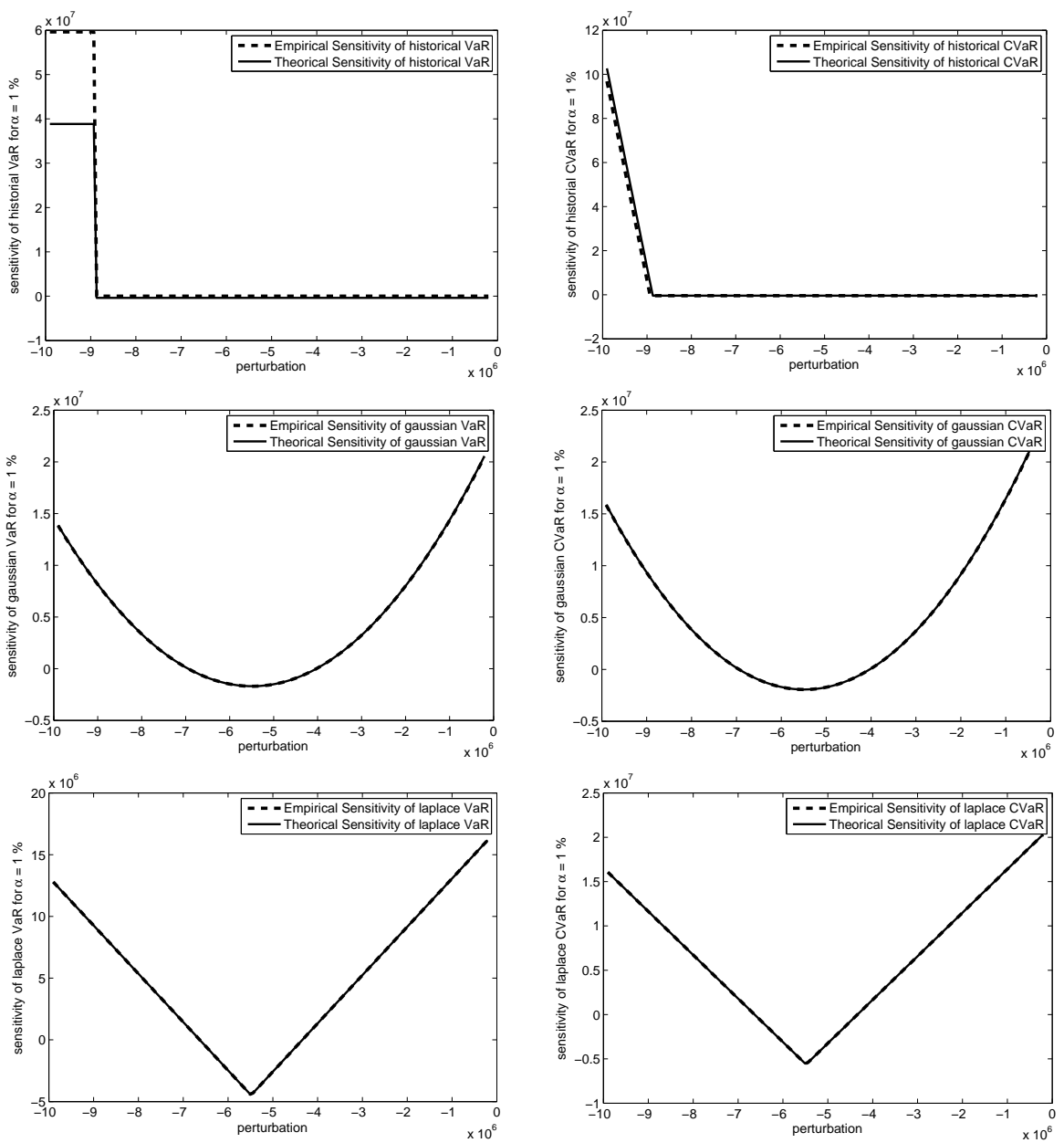

Figure 3: Empirical vs theoretical sensitivity functions of risk estimators for $\alpha=1 \%$ at a 1 day horizon. Historical VaR (upper left), Historical ES (upper right), Gaussian VaR (left), Gaussian ES (right), Laplace VaR (lower left), Laplace ES (lower right).

robustness and sensitivity properties of the risk estimators they use or design to assess the capital requirement, or manage their portfolio. Indeed, an unstable/ non-robust risk estimator, be it related to a coherent measure of risk, is of little use in practice.

Second, we have shown that the choice of the estimation method matters when discussing the robustness of risk measurement procedures: our examples show that different estimation methods coupled with the same risk measure lead to very different properties in terms of robustness and sensitivity. 
Historical VaR is a qualitatively robust estimation procedure, whereas the proposed examples of coherent (distribution-based) risk measures do not pass the test of qualitative robustness and show high sensitivity to "outliers". This explains perhaps why many practitioners have been reluctant to adopt "coherent" risk measures. Also, most parametric estimation procedures for VaR and ES lead to nonrobust estimators. On the other hand weighted averages of historical VaR like

$$
\frac{1}{\alpha_{2}-\alpha_{1}} \int_{\alpha_{1}}^{\alpha_{2}} \operatorname{VaR}_{u}^{h}(F) d u
$$

with $1>\alpha_{2}>\alpha_{1}>0$ have robust empirical estimators.

\subsection{Re-examining subadditivity}

The conflict we have noted between robustness of a risk measurement procedure and the subadditivity of the risk measure shows that one cannot achieve robust estimation in this framework while preserving subadditivity. While a strict adherence to the coherence axioms of Artzner et al [3] would push us to choose subadditivity over robustness, several recent studies $[5,12,14]$ have provided reasons for not doing so.

Danielsson et al. [5] explore the potential for violations of VaR subadditivity and report that for most practical applications VaR is sub-additive. They conclude that in practical situations there is no reason to choose a more complicated risk measure than VaR, solely for reasons of subadditivity. Arguing in a different direction, Ibragimov \& Walden [14] show that for very "heavy-tailed" risks defined in a very general sense, diversification does not necessarily decrease tail risk but actually can increase it, in which case requiring sub-additivity would in fact be unnatural. Finally, Heyde et al [12] argue against subadditivity from an axiomatic viewpoint and propose to replace it by a weaker property of co-monotonic subadditivity. All these objections to the sub-additivity axiom deserve serious consideration and further support the choice of robust risk measurement procedures over non-robust ones for the sole reason of saving subadditivity.

\subsection{Beyond distribution-based risk measures}

While the 'axiomatic' approach to risk measurement embodies in principle a much wider class of risk measures than distribution-based -or "law-invariant"risk measures, research has almost exclusively focused on this rather restrictive class of risk measures. Our result, that coherence and robustness cannot coexist within this class, can also be seen as an argument for going beyond (and abandoning) distribution-based risk measures. This also makes sense in the context of the ongoing discussion on systemic risk: evaluating exposure to systemic risk only makes sense in a framework where one considers the joint distribution of a portfolio's losses with other, external, risk factors, not just the marginal distribution of its losses. In fact, non-distribution-based risk measures are routinely used in practice: the Standard Portfolio Analysis of Risk (SPAN) 
margin system, used by several exchanges and cited as the original motivation in [3], is a well-known example of such a method.

We hope to have convinced the reader that there is more to risk measurement than the choice of a "risk measure". We think that the property of robustness - and not only the coherence - should be a concern for regulators and end-users when choosing or designing risk measurement procedures. What our study illustrates is that the design of robust risk estimation procedures requires the inclusion of the statistical estimation step in the risk measurement procedure. We hope this work will stimulate further discussion on these important issues.

\section{A Proofs}

\section{A.1 Proof of Proposition 2}

First, observe that for any fixed $\varepsilon>0$ and $G \in \mathcal{C}$, as $\widehat{\rho}^{h}$ is consistent with $\rho$ at $F$ and $G$, there exists $n^{*} \geq 1$ such that

$$
d\left(\mathcal{L}_{n}\left(\widehat{\rho}^{h}, F\right), \delta_{\rho(F)}\right)+d\left(\mathcal{L}_{n}\left(\widehat{\rho}^{h}, G\right), \delta_{\rho(G)}\right)<\frac{2 \varepsilon}{3}, \quad \forall n \geq n^{*} .
$$

"1. $\Rightarrow 2 "$. Assume that $\left.\rho\right|_{\mathcal{C}}$ is continuous at $F$ and fix $\varepsilon>0$. Then there exists $\delta>0$ such that if $d(F, G)<\delta$, then $d\left(\delta_{\rho(F)}, \delta_{\rho(G)}\right)=|\rho(F)-\rho(G)|<\varepsilon / 3$. Thus $\mathcal{C}$-robustness readily follows from $(22)$ and the triangular inequality

$$
\begin{aligned}
d\left(\mathcal{L}_{n}\left(\widehat{\rho}^{h}, F\right), \mathcal{L}_{n}\left(\hat{\rho}^{h}, G\right)\right) \leq \\
\quad \leq d\left(\mathcal{L}_{n}\left(\hat{\rho}^{h}, F\right), \delta_{\rho(F)}\right)+d\left(\delta_{\rho(F)}, \delta_{\rho(G)}\right)+\leq d\left(\mathcal{L}_{n}\left(\hat{\rho}^{h}, G\right), \delta_{\rho(G)}\right)
\end{aligned}
$$

$" 2$. $\Rightarrow 1 "$. Conversely, assume that $\hat{\rho}^{h}$ is $\mathcal{C}$-robust at $F$ and fix $\varepsilon>0$. Then there exists $\delta>0$ and $\bar{n} \geq 1$ such that

$$
d(F, G)<\delta, G \in \mathcal{C} \quad \Rightarrow \quad d\left(\mathcal{L}_{\bar{n}}\left(\widehat{\rho}^{h}, F\right), \mathcal{L}_{\bar{n}}\left(\widehat{\rho}^{h}, G\right)\right)<\varepsilon / 3
$$

As a consequence, from (22) and the triangular inequality

$$
\begin{aligned}
\mid \rho(F) & -\rho(G) \mid=d\left(\delta_{\rho(F)}, \delta_{\rho(G)}\right) \leq \\
& \leq d\left(\delta_{\rho(F)}, \mathcal{L}_{n}\left(\widehat{\rho}^{h}, F\right)\right)+d\left(\mathcal{L}_{n}\left(\widehat{\rho}^{h}, F\right), \mathcal{L}_{n}\left(\widehat{\rho}^{h}, G\right)\right)+d\left(\mathcal{L}_{n}\left(\widehat{\rho}^{h}, G\right), \delta_{\rho(G)}\right),
\end{aligned}
$$

it follows that $\left.\rho\right|_{\mathcal{C}}$ is continuous at $F$.

\section{A.2 Proof of Theorem 2}

We will show that the continuity problem of the scale function $\sigma: \mathcal{D}_{\psi} \rightarrow \mathbb{R}^{+}$ of portfolios $X$ can be reduced to the continuity (on a properly defined space) issue of the location function of portfolios $Y=\log \left(X^{2}\right)$. The change of variable here is made to use the results of Huber [13] about weak continuity of location parameters. The distribution $F$ can be seen as the distribution of a portfolio $X_{0}$ 
with $\mu\left(X_{0}\right)=0$ and $\sigma\left(X_{0}\right)=1$. Then, by setting $Y_{0}=\log \left(X_{0}^{2}\right)$, and denoting by $G$ the distribution of $Y_{0}$ we have:

$$
\begin{aligned}
G(y) & =P\left(Y_{0} \leq y\right)=P\left(X_{0}^{2} \leq e^{y}\right)=F\left(e^{y / 2}\right)-F\left(-e^{y / 2}\right) \\
g(y) & =G^{\prime}(y)=e^{y / 2} f\left(e^{y / 2}\right)
\end{aligned}
$$

Moreover, by introducing the following function:

$$
\varphi(y)=-\frac{g^{\prime}(y)}{g(y)} \quad \mathcal{D}_{\varphi} \triangleq\left\{G: \int \varphi(y) G(d y)<\infty\right\},
$$

we can define, as in Huber [13], the ML location function $\mu(H)$ for any distribution $H \in \mathcal{D}_{\varphi}$ as the solution of the following implicit relation

$$
\int \varphi(y-\mu) H(d y)=0 .
$$

Now, we consider the distribution $F_{X} \in \mathcal{D}_{\psi}$ of the random variable $X$ representing the P\&L of a portfolio and assume that $F_{X}$ has density $f_{X}$ and that the solution to $\int \psi\left(\frac{x}{\sigma}\right) F_{X}(d x)=0$ has a unique solution $\sigma=\sigma\left(F_{X}\right)$. Denoting by $F_{Y}$ the distribution of $Y=\log \left(X^{2}\right)$, it is easy to check that $F_{Y} \in \mathcal{D}_{\varphi}$ since, for $y=\log \left(x^{2}\right)$, we have:

$$
\begin{aligned}
\varphi(y) & =-\frac{g^{\prime}(y)}{g(y)} \\
& =-\frac{\frac{1}{2} e^{(y) / 2} f\left(e^{(y) / 2}\right)+\frac{1}{2} e^{y} f^{\prime}\left(e^{(y) / 2}\right)}{e^{(y) / 2} f\left(e^{(y) / 2}\right)} \\
& =-\frac{1}{2}\left[1+e^{(y) / 2} \frac{f^{\prime}\left(e^{(y) / 2}\right)}{f\left(e^{(y) / 2}\right)}\right] \\
& =-\frac{1}{2}\left[1+x \frac{f^{\prime}(x)}{f(x)}\right]=\frac{1}{2} \psi(x) .
\end{aligned}
$$

Noticing that

$$
F_{Y}(d y)=f_{Y}(y) d y=x f_{X}(x) d\left(\log \left(x^{2}\right)\right)=2 f_{X}(x) d x=2 F_{X}(d x),
$$

we immediately obtain from Equations (17), (23) and (24) that $\sigma\left(F_{X}\right)=\mu\left(F_{Y}\right)$ when $Y=2 \log (X)$. We have therefore shown that a scale function characterized by the function $\psi$, can also be interpreted as a location function characterized by the function $\varphi$. From Equation (24), we see that for all $x \in \mathbb{R}, 2 \varphi(x)=\psi\left[e^{x / 2}\right]$. Therefore, as $\psi$ is assumed to be even and increasing on $\mathbb{R}^{+}$, it implies that $\varphi$ is increasing on $\mathbb{R}$. Moreover, as $\psi$ takes values of both signs it is also true for $\varphi$. To conclude, we apply [13, Theorem 2.6] which states that a location function associated to $\varphi$ is weakly continuous at $G$ if and only if $\varphi$ is bounded and the location function computed at $G$ is unique. 


\section{References}

[1] C. ACERBI, Spectral measures of risk: a coherent representation of subjective risk aversion, Journal of Banking and Finance, 26 (2002), pp. 15051518.

[2] - Coherent measures of risk in everyday market practice, Quantitative Finance, 7 (2007), pp. 359-364.

[3] P. Artzner, F. Delbaen, J. Eber, and D. Heath, Coherent Measures of Risk, Mathematical Finance, 9 (1999), pp. 203-228.

[4] V. Costa and J. Deshayes, Comparaison des RLM estimateurs. Theorie de la robustesse et estimation d'un parametre, Asterisque, (1977), pp. 4344.

[5] J. Danielsson, B. Jorgensen, G. Samorodnitsky, M. SArma, And C. DE VRIES, Subadditivity re-examined: The case for Value-at-Risk, Preprint, London School of Economics, (2005).

[6] C. Deniau, G. Oppenheim, and C. Viano, Courbe d'influence et sensibilite. Theorie de la robustesse et estimation d'un parametre, Asterisque, (1977), pp. 43-44.

[7] H. Föllmer AND A. Schied, Convex measures of risk and trading constraints, Finance and Stochastics, 6 (2002), pp. 429-447.

[8] —, Stochastic Finance: An Introduction in Discrete Time, Walter de Gruyter, 2004.

[9] M. Frittelli and E. Rosazza Gianin, Putting order in risk measures, Journal of Banking and Finance, 26 (2002), pp. 1473-1486.

[10] C. Gourieroux AND W. Liu, Sensitivity analysis of distortion risk measures, working paper, 2006.

[11] F. Hampel, E. Ronchetti, P. Rousseeuw, and W. Stahel, Robust Statistics: The Approach Based on Influence Functions, New York, (1986).

[12] C. Heyde, S. Kou, And X. Peng, What Is a Good Risk Measure: Bridging the Gaps between Data, Coherent Risk Measures, and Insurance Risk Measures, Preprint, Columbia University, (2007).

[13] P. Huber, Robust Statistics, Wiley, 1981.

[14] R. Ibragimov And J. WaLden, The limits of diversification when losses may be large, Journal of Banking and Finance, 31 (2007), pp. 2551-2569.

[15] S. KusuokA, On law invariant coherent risk measures, Advances in Mathematical Economics, 3 (2001), pp. 83-95. 
[16] R. Rockafellar and S. Uryasev, Conditional Value-at-Risk for General Distributions, Journal of Banking and Finance, 26 (2002), pp. 14431471.

[17] D. Tasche, Expected Shortfall and Beyond, Statistical Data Analysis Based on the L1-Norm and Related Methods, (2002).

[18] W. Van Zwet, A strong law for linear functions of order statistics, Ann. Probab, 8 (1980), pp. 986-990. 